\title{
SISTEMA DE MEDIÇÃO DE PERFIL DE BARRAS FORJADAS BASEADO EM VISÃO COMPUTACIONAL*
}

Renato Ferreira Lima e Corrêa Pereira Luiz Fernando Etrusco Moreira ${ }^{2}$ Marcus Vinícius Gomes Tamietti ${ }^{3}$ Paulo Augusto Morais De Oliveira ${ }^{4}$ Marcos Roberto Mantovani Luiz Donizetti Gasparini ${ }^{6}$ Gerson Graciano ${ }^{7}$

\section{Resumo}

Na manufatura de produtos metálicos, o forjamento é o processo de transformação a quente de materiais dúcteis, como aços e ligas, através de conformação mecânica efetuada com esforço de compressão por uma prensa, de modo que eles assumam os formatos desejados. Este processo normalmente é utilizado para produzir peças de formas complexas e com uma maior precisão dimensional de acordo com a especificidade exigida pelo cliente final. Para obter resultados de qualidade, foi necessária a implantação de técnicas de medição indireta de última geração, que proporcionam ganho de tempo e a redução de desperdício de energia e material, além de aumentar a segurança, reduzindo a presença de pessoas próximas aos materiais para medições manuais. O presente trabalho trata da criação de um sistema de visão que, por meio de análise de imagem, é capaz de coletar a medida do perfil de barras em uma Forjaria em tempo real, podendo ser utilizado durante a operação da prensa, para garantir que o material está em conformidade com o especificado. A execução do sistema em produção aponta que as medidas realizadas apresentam desvio máximo de $2 \mathrm{~mm}$ em relação àquelas realizadas pelos operadores.

Palavras-chave: Visão computacional; Análise dimensional; Medição indireta; Forjamento.

\section{SYSTEM FOR MEASURING THE PROFILE OF FORGED PARTS USING COMPUTER VISION}

\section{Abstract}

Forging is a manufacturing process of hot transforming ductile materials like steel and alloys by mechanical shaping with compressive force using a press, so that they take on a desired form. This process is used to produce parts of complex shapes and greater dimensional accuracy in accordance with customers' specific requirements. In order to obtain high quality results, state-of-the-art techniques for indirect measurement were required, saving time, decreasing wasted energy and material, in addition to increasing safety and reducing the presence of people close to the parts for manual measurements. This work presents the creation of a vision system which, through image analysis, is able to perform the measurement of parts' profile in the Forge online, during the operation of the press, to ensure that the parts comply with their expected dimension. The execution of the system in production shows that the measurements had maximum deviation of $2 \mathrm{~mm}$ in comparison to those performed manually by the operators.

Keywords: Computer vision; Dimensional analysis; Indirect measurement; Forging.

1 Engenheiro de Computação, Gerente de Software, Invent Vision, Engenho Nogueira, MG, Brasil; renato.correa@ivision.ind.br.

2 Doutor em Engenharia Elétrica, Diretor de Tecnologia, Invent Vision, Engenho Nogueira, MG, Brasil, luizf@ivision.ind.br.

3 Eng. Eletricista, Gerente de Operações, Invent Vision, Engenho Nogueira, MG, Brasil; marcus@ivision.ind.br.

4 Engenheiro Metalurgista, Gerente de Setor, Forjaria, Villares Metals SA, Sumaré, SP, Brasil; paulo.morais@villaresmetals.com.br.

5 Tecnólogo em Instrumentação e Controle, Pós-graduação em Qualidade e Produtividade, Supervisor Lab. de Metrologia e Instrumentação, Villares Metals SA, Sumaré, SP, Brasil; marcos.mantovani@villaresmetals.com.br.

6 Bacharel em Direito, Metrologista III, Laboratório de Metrologia, Villares Metals SA, Sumaré, SP, Brasil; luiz.gasparini@villaresmetals.com.br.

7 Engenheiro Mecânico, Diretor Industrial, Forjados, Villares Metals SA, Sumaré, SP, Brasil; gerson.graciano@villaresmetals.com.br.

* Contribuição técnica ao $18^{\circ}$ Seminário de Automação e TI Industrial, 23 a 26 de setembro de 2014, São Paulo, SP, Brasil. 


\section{INTRODUÇÃO}

Na manufatura de produtos metálicos, o forjamento é o processo de transformar um material dúctil, através de conformação mecânica efetuada com esforço de compressão, de tal modo que ele assuma um formato desejado. Este processo normalmente é utilizado para produzir peças de formas complexas e com uma maior precisão dimensional de acordo com a especificidade exigida pelo cliente final.

Para obter os resultados exigidos, é necessária a implantação de técnicas de medição online de última geração, que proporcionam ganho de tempo e muitas vezes a redução de desperdício de energia e material, além de aumentar a segurança, reduzindo a presença de pessoas próximas às peças para medições manuais.

$\mathrm{Na}$ Prensa de 3000 t da Forjaria da Villares Metals o processo de medição dos materiais era sempre realizado manualmente por operadores por meio de compasso e escala graduada. Dessa forma, visando a aumentar a segurança dos operadores e a qualidade do produto, houve a necessidade de modernizar o processo, de modo que os seguintes objetivos fossem atendidos:

- Permitir adquirir a medida dos perfis das barras de forma indireta, rápida e remota, por comando do operador.

- Possibilitar detectar rapidamente condições anormais de operação na prensa, o que permite realizar ações corretivas imediatas.

- Proporcionar maior segurança na operação, reduzindo a presença frequente do operador em área de movimentação da prensa, manipuladores e dos materiais.

- Aumentar a agilidade no processo de medição.

- Tornar possível rastrear as medidas realizadas com as respectivas imagens capturadas.

Soluções que diminuam a intervenção humana sempre foram uma demanda muito comum em ambientes industriais. Sistemas baseados em visão computacional têm se tornado cada vez mais utilizados [1,4], uma vez que, além de garantir mais robustez e repetitividade [2,3], diminuem a chance de acidentes em ambientes críticos.

Este trabalho apresenta um sistema industrial de medição por análise de imagem instalado na Prensa de 3000 t na Forjaria da Villares Metals, capaz de coletar a medida do perfil de barras forjadas em tempo real, durante a operação da prensa.

\section{MATERIAIS E MÉTODOS}

A implantação de um sistema de visão computacional requer o entendimento dos componentes desse sistema. Esses componentes, apresentados na Figura 1, serão descritos a seguir.

\subsection{Componentes de um Sistema de Visão}

Os principais componentes de um Sistema de Visão são:

- $\quad$ Câmeras: As câmeras são os dispositivos responsáveis por capturar imagens dos objetos ou regiões inspecionados. A escolha da câmera ideal requer a definição cuidadosa de parâmetros tais como resolução, sensibilidade, velocidade de aquisição e interfaces padrão.

- Ótica: A escolha das lentes e filtros deve levar em consideração a distância focal e a distância de trabalho, de forma a permitir a captura de imagens ideais.

* Contribuição técnica ao $18^{\circ}$ Seminário de Automação e TI Industrial, 23 a 26 de setembro de 2014, São Paulo, SP, Brasil. 
Lentes inadequadas podem ocasionar imagens com distorção além do tolerado, o que dificulta os métodos de visão computacional.

- Sistema de lluminação: Nos ambientes de siderurgia, a variação de luminosidade pode ser brusca devido à presença de elementos incandescentes causados, por exemplo, pelo carregamento de ferro gusa em uma panela. Dentre as opções de iluminação podem-se citar as lâmpadas fluorescentes e halógenas, além de iluminação por leds.

- Software: O software é a alma do sistema de visão e pode ser executado pelo computador ou por um processador embutido na câmera, no caso das chamadas Smart Cameras.

- Unidade de Interface - IHM e Unidade de E/S: Os sistemas de visão possuem algumas interfaces principais, além da interface com a câmera: a interface com o operador e a interface com a automação. A interface com o operador do sistema normalmente é composta por um sinótico simplificado que mostra ao operador questões críticas do teste ou do status do sistema, permitindo a rastreabilidade das peças e sinais luminosos ou sonoros indicando alguma condição de alerta aos operadores. Já a interface com a automação envolve comunicação via portas serial e/ou ethernet, ou qualquer outro meio de comunicação do computador com um hardware externo capaz de transmitir sinais de controle para a automação.

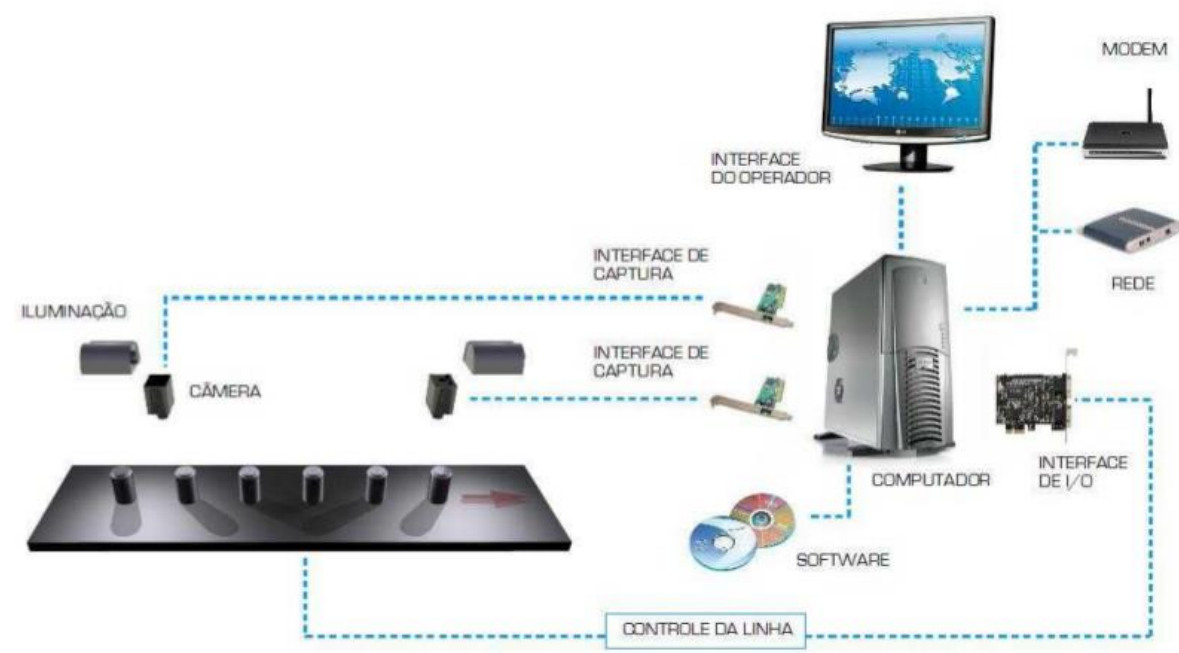

Figura 1. Exemplo típico de arquitetura de um sistema de visão.

\subsection{Procedimentos e Configuração dos Equipamentos Utilizados no Trabalho}

O sistema foi ajustado para funcionamento na Prensa de 3000 t na Forjaria da Villares Metals. A faixa de diâmetros de peças produzidas nessa prensa varia entre $165 \mathrm{~mm}$ e $600 \mathrm{~mm}$. As peças sempre são forjadas apoiadas sobre uma bigorna.

Além disso, as peças a serem medidas encontram-se numa faixa de temperatura entre $800^{\circ} \mathrm{C}$ e $1000^{\circ} \mathrm{C}$, dado importante para se definir a faixa de tempos de exposição durante a captura de imagens. A tabela 1 reúne as características das peças a serem medidas pelo sistema.

A câmera foi instalada dentro da sala de controle, a uma distância de aproximadamente $5 \mathrm{~m}$ do centro da prensa.

* Contribuição técnica ao $18^{\circ}$ Seminário de Automação e TI Industrial, 23 a 26 de setembro de 2014, São Paulo, SP, Brasil. 


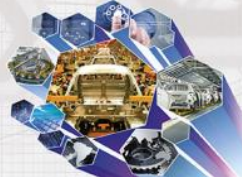

Tabela 1. Características das peças a serem medidas

\begin{tabular}{ccccc}
$\begin{array}{c}\text { Formato das } \\
\text { Peças }\end{array}$ & $\begin{array}{c}\text { Menor } \\
\text { diâmetro }\end{array}$ & $\begin{array}{c}\text { Maior } \\
\text { diâmetro }\end{array}$ & $\begin{array}{c}\text { Temperatura } \\
\text { mínima }\end{array}$ & $\begin{array}{c}\text { Temperatura } \\
\text { máxima }\end{array}$ \\
\cline { 1 - 3 } Cilíndrico & $165 \mathrm{~mm}$ & $600 \mathrm{~mm}$ & $800^{\circ} \mathrm{C}$ & $1000^{\circ} \mathrm{C}$
\end{tabular}

Um parâmetro importante para a realização de análise dimensional por imagens é a obtenção da resolução espacial do sistema que é dada em $\mathrm{mm} /$ pixel. Definidas as dimensões do alvo que se deseja medir, esse parâmetro é obtido considerando-se basicamente a resolução oferecida pela câmera, a distância até o alvo e a lente utilizada.

A técnica desenvolvida deve ser suficientemente acurada e precisa, de modo que, com a resolução espacial obtida, o sistema seja capaz de realizar medições dentro da faixa de erro esperada.

A tabela 2 resume os parâmetros de configuração ótica utilizada no sistema:

Tabela 2. Configuração ótica utilizada pelo sistema

\begin{tabular}{rll}
\hline Câmera & v200E \\
\hline Gesolução da imagem & $1944 \times 2592$ pixels \\
\hline Ganho & 1 \\
\hline Tempo de exposição & Entre $5 \mathrm{~ms}$ e $30 \mathrm{~ms}$ \\
\hline Abertura da Lente & $16 \mathrm{~mm}$ \\
\hline Íris & $\mathrm{f8,0}$ \\
\hline Ajuste de foco da lente & $\sim 1,0 \mathrm{~m}$ \\
\hline Campo de visão horizontal & $\sim 12^{\circ}$ \\
\hline Campo de visão vertical & $\sim 16^{\circ}$
\end{tabular}

A figura 2 apresenta a disposição dos equipamentos envolvidos no sistema.

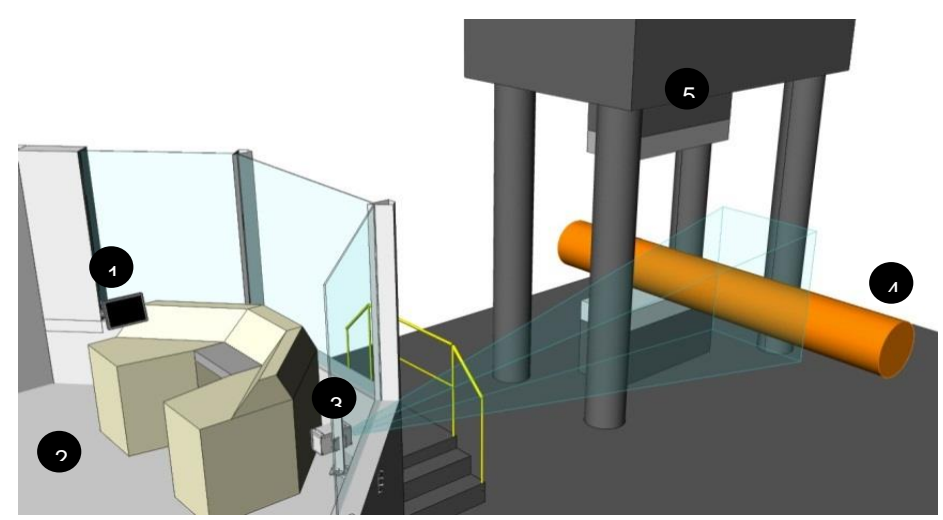

(a)

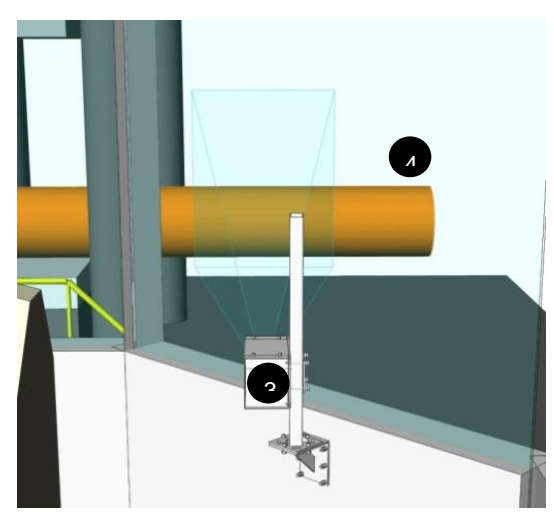

(b)

Figura 2. Disposição dos equipamentos envolvidos no sistema. (a) Visão de fora da cabine de comando. (b) Visão do local onde a câmera foi posicionada. 1 - Computador e interface do sistema com o operador; 2 - Cabine de comando do operador; 3 - Case da câmera; 4 - Material a ser forjado; 5 - Prensa.

Nas seções a seguir será apresentada a metodologia desenvolvida para a medição do perfil de barras.

\subsection{Calibração do Sistema}

Inicialmente foi realizada a calibração da câmera [5], visando a obter seus parâmetros intrínsecos e os coeficientes de distorção. Em seguida, mediu-se a

* Contribuição técnica ao $18^{\circ}$ Seminário de Automação e TI Industrial, 23 a 26 de setembro de 2014, São Paulo, SP, Brasil. 
distância entre o flange da câmera e o centro da bigorna com o auxílio de uma trena a Laser de alta precisão.

Para forjar peças de formato cilíndrico, a bigorna tipo "V" é utilizada. O uso dessa bigorna faz com que o plano de medição esteja sempre a uma distância I do flange da câmera, independente do raio do cilindro, como mostra a figura 3.

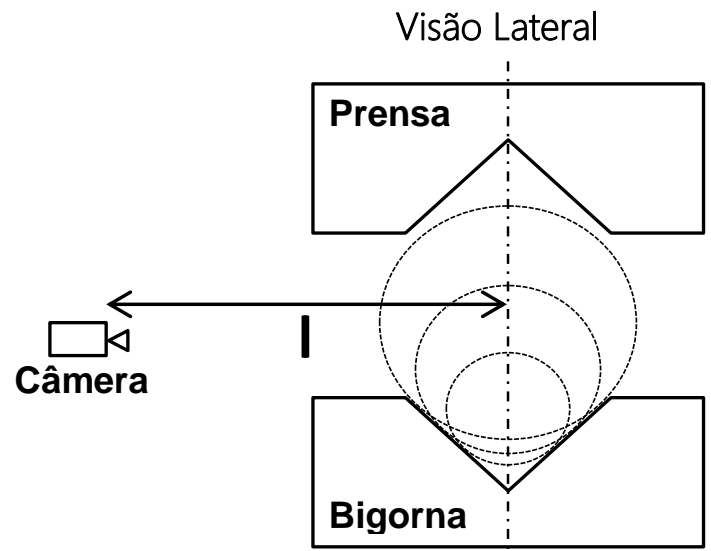

Figura 3. Visão lateral da cena. O flange da câmera está sempre a uma distância I do centro da bigorna tipo "V".

\subsection{Método de Medição do Perfil de Barras}

Realizada a etapa de calibração, a primeira etapa para a medição do perfil das barras é a aquisição da imagem. A imagem típica de uma peça capturada durante o forjamento é apresentada na figura 4.

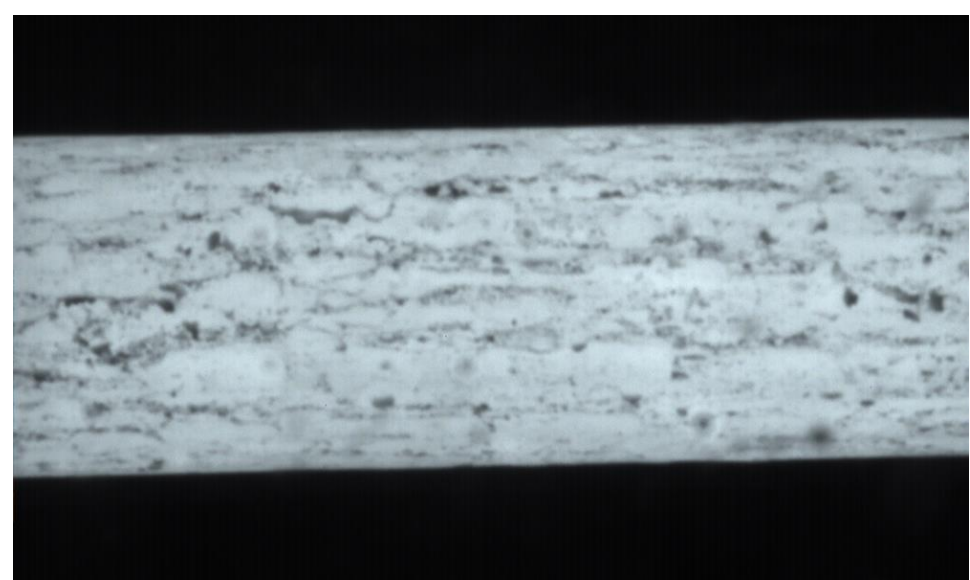

Figura 4. Imagem típica capturada de peças durante o forjamento.

A temperatura da barra influencia diretamente a intensidade dos pixels que a representam na imagem: fixando todos os parâmetros de captura (sobretudo o tempo de exposição), barras mais quentes apresentam uma média de intensidade de pixels mais alta (mais próxima do branco), enquanto e barras mais frias apresentam intensidade mais baixa. Para que a variação de temperatura da barra não influencie na qualidade da medição, após a captura aplica-se um algoritmo que determina a intensidade média dos pixels da barra. Estando a média fora de uma faixa determinada de valores, realizam-se capturas sucessivas, variando-se o tempo de exposição conforme a necessidade de aumentar ou diminuir a intensidade média dos pixels.

* Contribuição técnica ao $18^{\circ}$ Seminário de Automação e TI Industrial, 23 a 26 de setembro de 2014, São Paulo, SP, Brasil. 
Encontrada a imagem ideal, remove-se a distorção esférica da imagem por meio dos coeficientes obtidos na etapa de calibração. Em seguida, aplica-se um filtro de mediana de kernel suficientemente pequeno visando a eliminar pequenos ruídos sem alterar as bordas da imagem.

A partir de uma coordenada do eixo horizontal da imagem, escolhida através de interface com o operador, realizam-se buscas pelas fronteiras superior e inferior da barra. Encontrados os pontos limites da barra no sistema de coordenadas da imagem (em duas dimensões), o próximo passo consiste em determinar a localização desses pontos no mundo (em três dimensões). Considerando que a câmera é a origem do mundo, através dos parâmetros intrínsecos obtidos na calibração, e conhecendo o valor I que representa a distância entre o flange da câmera e o plano de medição, obtém-se a representação tridimensional dos pontos da fronteira da barra através da relação descrita na equação (1):

$$
\begin{aligned}
& \begin{array}{lll}
P & Q & W
\end{array} \\
& {\left[\begin{array}{c}
x_{p} \\
y_{p} \\
1
\end{array}\right]=\left[\begin{array}{cccc}
f_{x} & 0 & c_{x} & 0 \\
0 & f_{y} & c_{y} & 0 \\
0 & 0 & 0 & 1
\end{array}\right]\left[\begin{array}{c}
X_{w} \\
Y_{w} \\
Z_{w} \\
1
\end{array}\right] \quad \therefore \quad \begin{array}{r}
P=Q W \\
Q^{\dagger} P=Q^{\dagger} Q W \\
\end{array}}
\end{aligned}
$$

Na relação acima, $P$ é um ponto na imagem, $Q$ é a matriz da câmera $\left(Q^{\dagger}\right.$ é sua pseudoinversa), contendo seus parâmetros intrínsecos e $W$ é um ponto no mundo. A equação 1 mostra que um ponto na imagem corresponde a uma reta no mundo real. Para obter as coordenadas de um ponto no mundo real ao longo dessa reta, é necessário encontrar o plano em que ela é interceptada. Considerando que o plano de medição é ortogonal ao plano XY da câmera e está localizado a uma distância I de seu flange, obtêm-se os valores das coordenadas da fronteira no mundo (tridimensional).

A figura 5 mostra os pontos $\mathrm{T}$ e B encontrados após os passos descritos acima.

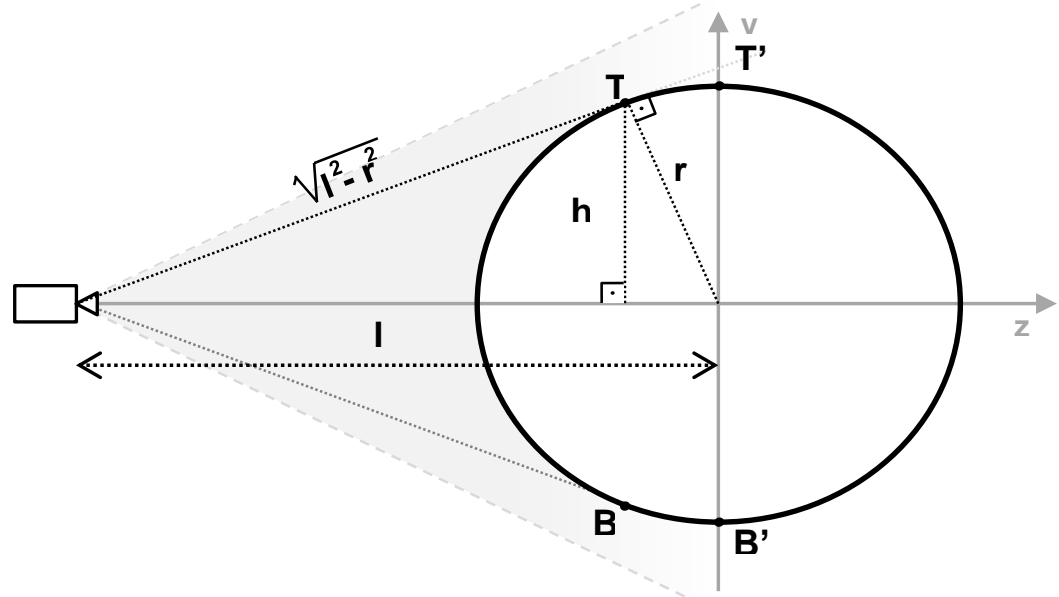

Figura 5. Visão lateral da cena: os pontos T e B não correspondem às extremidades da barra.

Os pontos $\mathrm{T}$ e $\mathrm{B}$ encontrados no algoritmo descrito até o presente ponto não correspondem às extremidades da barra. Ainda observando a figura 5, é possível extrair a seguinte relação:

$$
\frac{h}{\sqrt{l^{2}-r^{2}}}=\frac{r}{l}
$$

$$
\text { Isolando } r \text {, temos: } r=l \sqrt{\frac{1-\sqrt{1-\frac{4 h^{2}}{l^{2}}}}{2}}
$$

* Contribuição técnica ao $18^{\circ}$ Seminário de Automação e TI Industrial, 23 a 26 de setembro de 2014, São Paulo, SP, Brasil. 


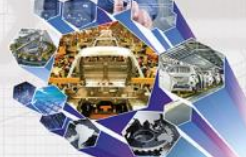

Com o valor do raio $r$, obtêm-se as extremidades da barra e, então, seu diâmetro.

\section{RESULTADOS E DISCUSSÃO}

Após instalação do sistema em linha, a câmera foi configurada com os parâmetros óticos adequados, o sistema foi calibrado e diversas medições foram feitas, tanto manualmente pelos operadores, quanto remotamente, pelo sistema construído.

Ao longo de várias semanas, toda peça forjada na Prensa de 3000 t com formato cilíndrico fora medida tanto pelo sistema desenvolvido, quanto pelos operadores, manualmente. As peças possuíam as dimensões e temperaturas especificadas na tabela 1 da seção 2.2. Para avaliar o sistema desenvolvido, foi realizada uma análise estatística das variáveis envolvidas com 82 amostras.

As medidas informadas pelo sistema desenvolvido (i), pelo operador (ii) e pela programação do equipamento (iii), foram registradas em uma planilha, junto à diferença entre as duas medições (i-ii), ao nome do operador, e à temperatura no momento da medição, no formato da figura 6 . Em seguida, procedeu-se com a análise das variáveis envolvidas.

\begin{tabular}{|c|c|c|c|c|c|c|c|}
\hline Data & $\begin{array}{c}\mathbf{i}-\text { Medição do } \\
\text { Sistema (mm) }\end{array}$ & $\begin{array}{c}\text { ii }- \text { Medição do } \\
\text { Operador }(\mathbf{m m})\end{array}$ & $\begin{array}{c}\text { iii - Bitola } \\
\text { Programada }(\mathbf{m m})\end{array}$ & $\begin{array}{c}\text { Diferença } \\
(\mathbf{i}-\mathbf{i i})\end{array}$ & $\begin{array}{c}\text { Temperatura } \\
\left({ }^{\circ} \mathbf{c}\right)\end{array}$ & Operador & Hora \\
\hline $13 / 11 / 13$ & 218 & 220 & 216 & -2 & 790 & 1 & $00: 01$ \\
\hline $14 / 11 / 13$ & 290 & 288 & 288 & 2 & 950 & 2 & $07: 08$ \\
\hline$\vdots$ & $\vdots$ & $\vdots$ & $\vdots$ & $\vdots$ & $\vdots$ & $\vdots$ & $\vdots$ \\
\hline
\end{tabular}

Figura 6. Exemplo da planilha de registro das medições.

\subsection{Análise de Correlação entre as Medidas Realizadas}

Para se verificar a correlação entre as variáveis: (i) medida realizada pelo sistema desenvolvido e (ii) medida realizada pelos operadores, foi feita uma análise de regressão (linear simples), como na figura 7 . Revelou-se correlação total entre elas.

Análise de Regressão:

(i) Diâmetro Sistema x (ii) Diâmetro Manual

Equação de regressão:

(i) $=1,34+0,993$ (ii)

$\begin{array}{lll}\text { Predictor } & \text { Constant } & (\text { ii) } \\ \text { Coef } & 1,34 & 0,993 \\ \text { SE Coef } & 0,65 & 0,00242 \\ \text { T } & 2,07 & 410,56 \\ \text { P } & 0,042 & 0,000 \\ & \\ S=1,39947 & \\ \text { R-Sq }=100,0 \% & \\ \text { R-Sq(adj) }=100,0 \% & \end{array}$

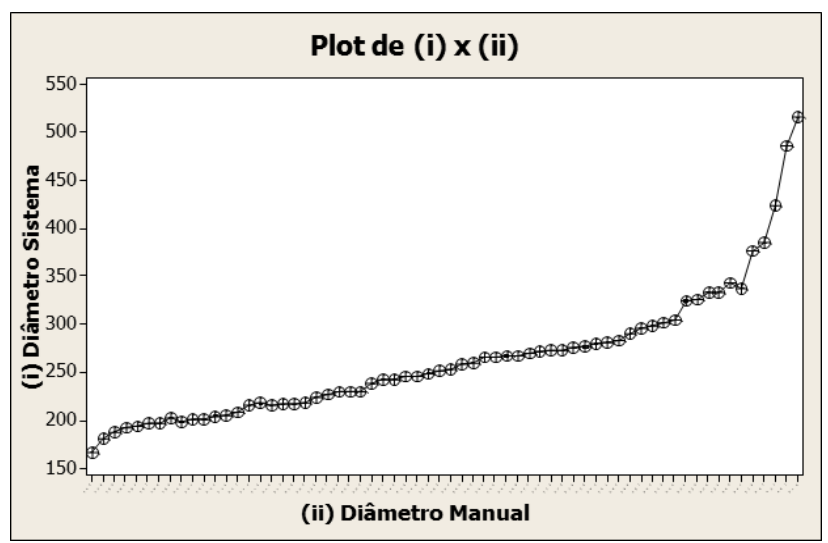

Figura 7. Resultado da análise de correlação entre as medidas realizadas pelo sistema e pelos operadores.

\subsection{Comparação das Diferenças entre as Medidas}

Para estimar o erro das medições realizadas pelo sistema desenvolvido, seria necessário obter as medidas reais das amostras. As únicas medidas disponíveis para comparação após o forjamento são:

* Contribuição técnica ao $18^{\circ}$ Seminário de Automação e TI Industrial, 23 a 26 de setembro de 2014, São Paulo, SP, Brasil. 
- A medição informada pelos operadores, que possui um erro associado, não sendo possível, portanto, adotá-la como sendo real.

- A medição informada na programação da prensa. Essa medida representa, na verdade, o valor da distância entre a base da bigorna e o topo do ferramental acoplado à prensa, utilizado para dar o formato desejado à barra. Essa medida também não pode ser adotada como real, uma vez que, com o passar do tempo, há um desgaste do ferramental.

Sendo assim, decidiu-se comparar as diferenças entre as três variáveis envolvidas: medida informada (i) pelo sistema desenvolvido, (ii) pelo operador e (iii) pela programação da prensa. Essa comparação tem como objetivo explicitar o valor central da diferença entre as medições e a dispersão em torno dele. A ferramenta que permite conhecer essas características em uma determinada distribuição é o histograma.

A figura 8 mostra o histograma que confronta a dispersão das diferenças de medições.
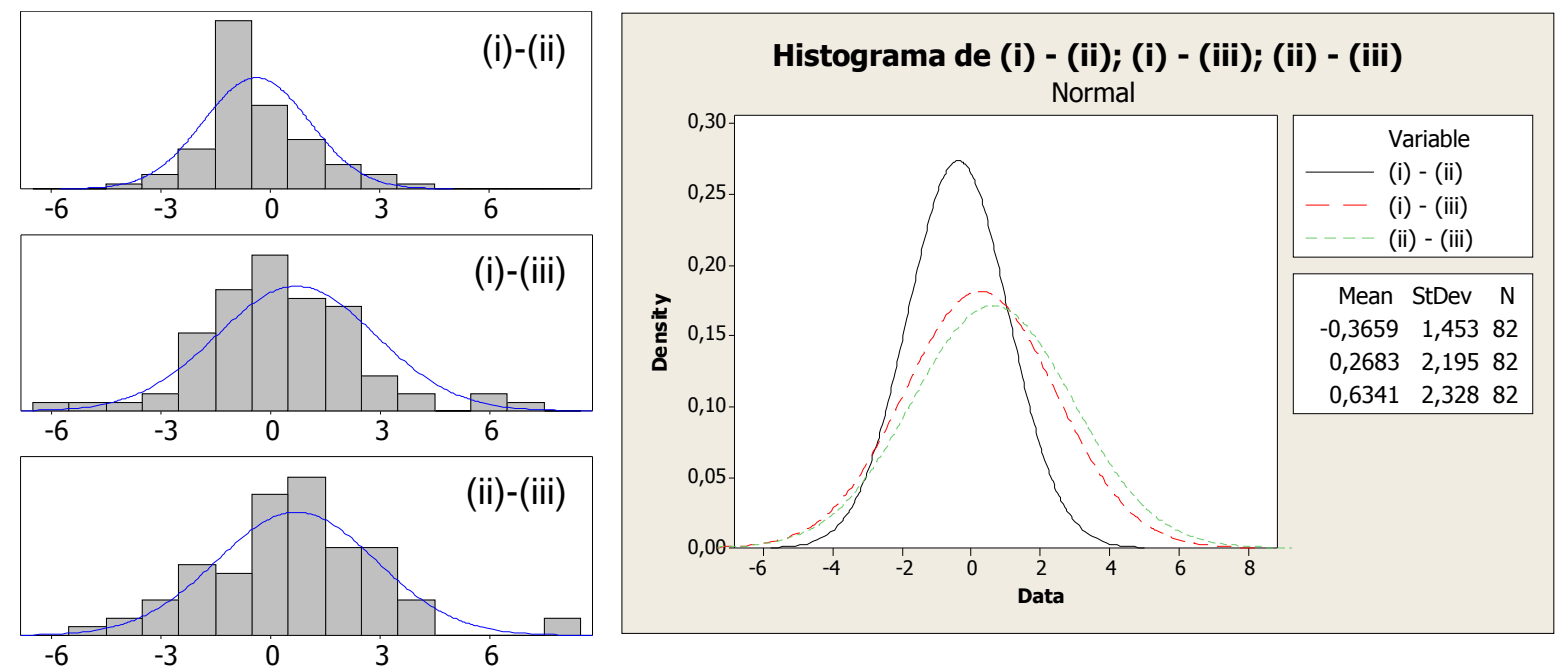

Figura 8. Histograma da diferença entre as medições.

Através dos gráficos da figura 8 , os seguintes resultados podem ser enumerados:

(a) A diferença entre a medição realizada pelo sistema é, em geral, menor que a realizada pelos operadores e concentra-se na faixa de $0 \mathrm{~mm}$ a $-1 \mathrm{~mm}$ : em média $0,37 \mathrm{~mm}$ menor.

(b) A diferença entre a medição realizada pelo sistema e a bitola informada pela programação da prensa é um pouco mais dispersa, mas concentra-se na faixa de $0 \mathrm{~mm}$ a $1 \mathrm{~mm}$ : em média, 0,27mm maior.

(c) Há uma diferença notável, também, entre as medidas realizadas pelos operadores e a bitola informada na programação da prensa. Apesar de apresentar dispersão similar à (i)-(iii), a diferença também se concentra na faixa de $0 \mathrm{~mm}$ a $-1 \mathrm{~mm}$, mas em um valor central um pouco maior: 0,63mm.

\subsection{Relação da Diferença entre as Medidas e Temperatura do Material}

Foi avaliado se existe relação entre a temperatura da barra e a diferença entre a medição pelo sistema e a realizada manualmente pelos operadores. Para tanto, foi disposto em um diagrama de dispersão o relacionamento entre as duas variáveis, como na figura 9.

* Contribuição técnica ao $18^{\circ}$ Seminário de Automação e TI Industrial, 23 a 26 de setembro de 2014, São Paulo, SP, Brasil. 


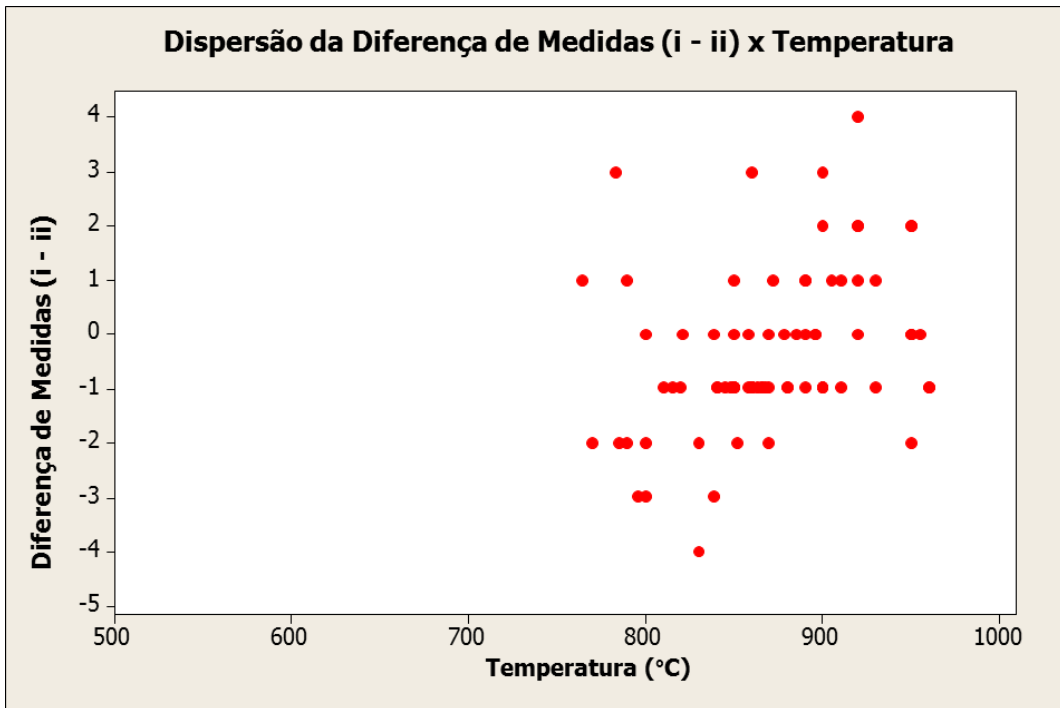

Figura 9. Diagrama de dispersão da diferença de medidas e da temperatura do material forjado. O diagrama de dispersão pode ser interpretado como mostra a figura 10.

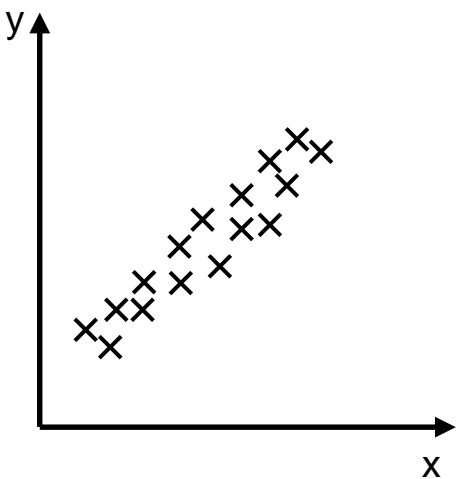

(a)

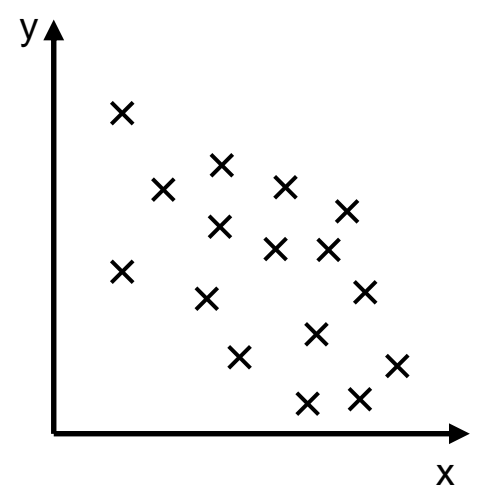

(d)

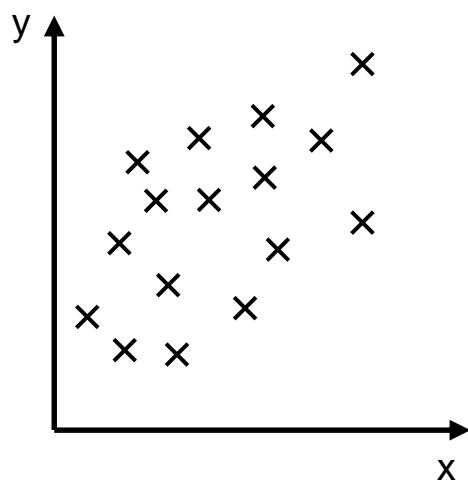

(b)

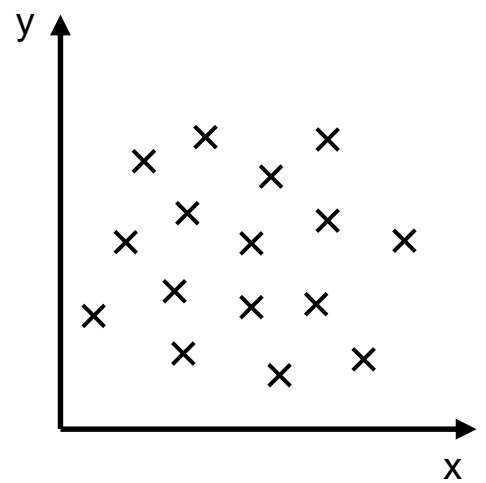

(c)

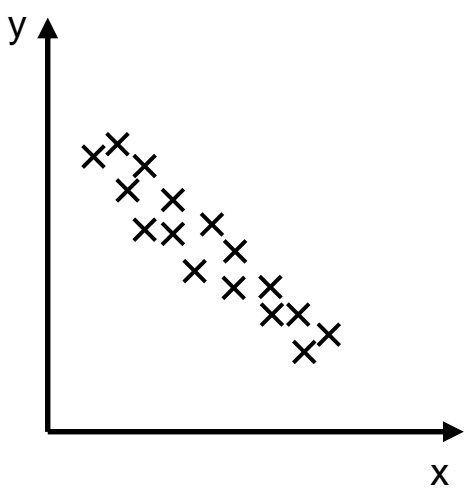

Legenda:

(a) elevada correlação positiva

(b) moderada correlação positiva

(c) ausência de correlação

(d) moderada correlação negativa

(a) elevada correlação negativa

(e)

Figura 10. Diagrama de dispersão da diferença de medidas e da temperatura do material forjado.

Assim, a figura 9 aponta ausência de relação entre a temperatura da barra e a diferença entre a medição do sistema e a realizada manualmente pelos operadores.

\section{CONCLUSÃo}

Os padrões de qualidade da Villares Metals definem que o instrumento de medição utilizado na Forjaria apresente erro máximo de $2 \mathrm{~mm}$. Após desenvolver análise 
estatística apurada, concluiu-se que a diferença entre as medições manual e pelo sistema desenvolvido concentra-se entre $0 \mathrm{~mm}$ e $-1 \mathrm{~mm}$.

Concluiu-se, também, que não existe influência expressiva da temperatura nas medições realizadas, seja com o sistema desenvolvido, seja com o método de medição manual.

Baseando na análise estatística, as medições realizadas pelo sistema desenvolvido não apresentam diferenças relevantes quando comparadas as medições manuais e, dessa forma, o sistema de medição desenvolvido atende a medição de barras cilíndricas na Forjaria.

\subsection{Trabalhos Futuros}

O sistema abordado neste trabalho foi concebido para realizar a medição de tubos de formato cilíndrico apenas. Na Villares Metals, peças de secção retangular (inclusive quadrada) também são forjadas, apesar de em menor quantidade, comparadas às de seção circular. Como mostra a figura 11, enquanto as peças cilíndricas sempre são medidas no mesmo plano de medição, as peças com diferentes formatos devem ser medidos em outros planos.

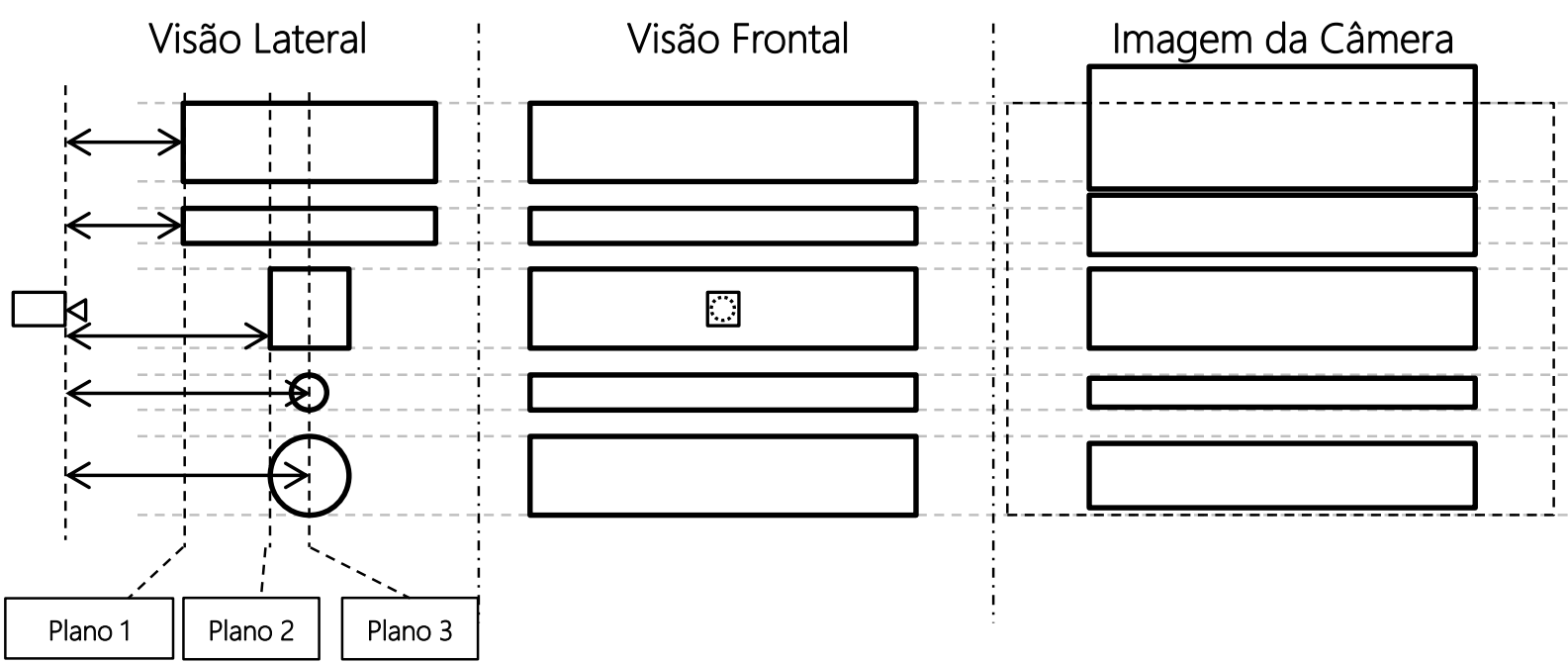

Figura 11. Visões lateral e frontal da cena, comparadas com uma possível representação na imagem. A diferença de profundidade entre os perfis dos objetos faz com que não seja possível medi-los pela imagem utilizando uma mesma calibração de plano de medição.

Devido a restrições no processo, não é possível posicionar com precisão adequada barras com formatos não cilíndricos, de modo que a face a ser medida fique sempre no mesmo plano, como mostra a figura 12.

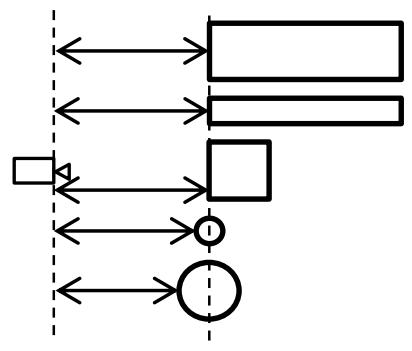

Figura 12. Visão lateral com todos os perfis no mesmo plano de medição.

* Contribuição técnica ao $18^{\circ}$ Seminário de Automação e TI Industrial, 23 a 26 de setembro de 2014, São Paulo, SP, Brasil. 
Dessa forma, espera-se que, futuramente, seja possível medir os outros formatos de barras, com o auxílio de um dispositivo capaz de fornecer a distância da câmera ao plano a ser medido, fazendo com que o sistema consiga corrigir o plano calibrado e medir a face em diferentes distâncias em relação à câmera.

\section{REFERÊNCIAS}

1 Moreira LFE, Oliveira CAA, Soares MB, Silva E. Sistema de tratamento e processamento de imagens: sistemas de visão no processo produtivo. Revista InTech América do Sul, n. 120, p. 32-39. ISSN: 1518-6024.

2 Reis LMSM, Moreira LFE, Soares MB. Reconhecimento automático do número da panela por visão computacional para tracking de panelas em aciarias. In: 15응 Seminário de Automação e TI Industrial, 2011, São Paulo/SP, Brasil.

3 Machado HN, Silva ED, Tamietti MVG, Moreira LFE, Oliveira M, Quintão I. Reconhecimento de texto impresso em chapas através de OCR. In: 15ํㅗㄴ Seminário de Automação e TI Industrial, 2011, São Paulo/SP, Brasil.

4 Soares MB, Moreira LFE, Diniz LFM, Neves AR. Sistemas para automatização de processos por meio de visão computacional nas indústrias siderúrgicas e metalúrgicas. In: 16 Seminário de Automação e TI Industrial, 2012, Belo Horizonte/MG, Brasil.

5 Zhang. A Flexible New Technique for Camera Calibration. IEEE Transactions on Pattern Analysis and Machine Intelligence, 2000; 22(11):1330-1334. 